\title{
LMS SaaS: Una alternativa para la formación virtual
}

\author{
SaaS LMS: An alternative to the virtual training \\ Jhon Francined Herrera-Cubides ${ }^{1 *} \quad$ Nancy Yaneth Gelvez-García ${ }^{1}$ \\ Danilo Alfonso López-Sarmiento ${ }^{1}$ \\ Recibido 23 de febrero de 2017, aceptado 19 de marzo de 2018 \\ Received: February 23, 2017 Accepted: March 19, 2018
}

\begin{abstract}
RESUMEN
Un programa de e-learning exitoso depende del LMS, acorde a las necesidades organizacionales específicas. El principal obstáculo que enfrentan muchas organizaciones es la inversión inicial que implica un LMS. $\mathrm{Al}$ incorporar un entorno de e-learning en su lugar de trabajo, es importante elegir un LMS construido como una solución SaaS, el cual permite adaptar sus características a las requerimientos solicitados, haciéndolo fácil de manejar y requiriendo poca administración de su parte.

En el marco del proyecto de diseño de una línea de producción tecnológica de objetos virtuales de aprendizaje, este artículo presenta una exploración acerca la alternativa de usar SaaS LMS como alternativa de gestión en e-learning, explorando características que proporciona un SaaS LMS. Para tales efectos se estudiará el desarrollo de aplicaciones en Cloud Computing, haciendo una caracterización general de SaaS y LMS, analizando los tipos de LMS y la interoperabilidad de aplicaciones y LMS, el trabajo a seguir y las conclusiones.
\end{abstract}

Palabras clave: Computación en la nube, SaaS, LMS, software como servicio, modelo de servicio, e-learning, sistema administrador de conocimiento.

\section{ABSTRACT}

An e-learning program success depends on the LMS, according to specific organizational needs. The main obstacle facing many organizations is the initial investment involving an LMS.

By incorporating an environment of e-learning in the workplace, it is important to choose a LMS built as a SaaS solution, which allows adapting its characteristics to the requested requirements, making it easy to handle and requiring little administrationt.

Under the project "Design of a technological production line of virtual learning objects", this article presents an exploration about the alternative of using SaaS LMS as alternative management in e-learning, exploring features that provides a SaaS LMS. For such purposes the development of Cloud computing applications will be studied, making a general characterization of SaaS and LMS, analyzing the types of LMS and interoperability of applications and LMS, work to follow and conclusions.

Keywords: Cloud computing, LMS, SaaS, service model, e-learning, learning management system.

\footnotetext{
1 Facultad de Ingeniería. Universidad Distrital Francisco José de Caldas. Bogotá, Colombia. E-mail: jfherrerac@udistrital.edu. co; nygelvezg@udistrital.edu.co; dalopezs@udistrital.edu.co
} 


\section{INTRODUCCIÓN}

Los procesos de formación han ido involucrado nuevas herramientas tecnológicas en su quehacer. Tal es el caso del e-learning, el cual se ha insertado en los procesos de formación como apoyo a la presencialidad (Blended Learning), o como estrategia completamente en línea. Para el desarrollo de un proceso de formación que vincule componentes de e-learning, se requiere de una plataforma de software especializada, denominada Learning Management System - LMS. Esta plataforma integra funcionalidades básicas como la gestión de cursos, publicación de contenidos, gestión de alumnos, sistemas de comunicación y sistemas de evaluación.

Regularmente los LMS usan modelos tradicionales de instalación, en el cual el aplicativo se ejecuta en los servidores del cliente. Para su adecuado funcionamiento, el equipo de Tecnologías de la Información (TI) del cliente, debe ofrecer el soporte, almacenamiento, redes y recursos necesarios para la operación del LMS.

Por otro lado, muchas organizaciones optan por adquirir LMS bajo un modelo SaaS - "Software as a Service", los cuales son gestionados en la nube. El modelo SaaS implica un alquiler de servicios, a través del pago en función de los recursos consumidos o de la potencial cobertura de los mismos. Los LMS SaaS proveen un elevado número de funcionalidades, y su mantenimiento y actualización está a cargo del proveedor de este servicio.

Bajo este contexto, el presente artículo se centra en desarrollar una exploración acerca de la conceptualización de LMS SaaS, sus características, tipos e interoperabilidad de aplicaciones. Para ello se parte de una revisión de los antecedentes. Posteriormente, se revisa el desarrollo de aplicaciones en Cloud Computing, como base de los modelos de servicios SaaS. Seguidamente se presentan el concepto de SaaS, LMS y sus diferentes tipos. A continuación, se presentan aspectos generales de la interoperabilidad de las aplicaciones y los LMS. Finalmente, se presentan los trabajos futuros y las conclusiones del proyecto de investigación en su estado actual.

\section{ANTECEDENTES}

Las organizaciones que han vinculado componentes de e-learning a los procesos de formación, han experimentado cómo sus sistemas de gestión académica funcionan de manera independiente a los sistemas de gestión de aprendizaje. Esta independencia les ha obligado a duplicar sus tareas, alimentando y gestionando cada sistema de forma separada. Un ejemplo de ello se observa en la creación de los cursos y la asignación de los estudiantes a los mismos. La inscripción de estudiantes debía realizarse previamente en el sistema de gestión académica, en los periodos de registro o matricula; posteriormente se debía crear manualmente los espacios académicos virtuales, y por último, se debía realizar la asignación de estudiantes a los citados cursos.

Adicionalmente, el hecho de gestionar una plataforma de LMS de manera local, involucra una serie de recursos tecnológicos, operativos y administrativos, requeridos para mantener funcionando adecuadamente el LMS. En términos generales, se debe contar un equipo de TI que se encargue de la instalación, configuración y adaptación del LMS. Además, se debe contar con una infraestructura tecnológica "in house", tales como máquinas, espacio de almacenamiento, funcionalidades de red, de seguridad, etc., necesarias para la administración del LMS.

La instalación "in house" del LMS implica necesariamente una inversión de dinero importante, y sobre todo implica un gasto fijo anual. Es claro, especialmente para las personas que han tenido que dirigir estos procesos y que son responsables de los mismos, que la flexibilidad de la infraestructura que soporta el LMS es mínima. A la hora de renovar la infraestructura, de incorporar nuevos elementos que la enriquezcan y que permitan nuevas adaptaciones metodológicas, derivadas de los avances en la tecnología y de las propias teorías educativas; conlleva un consumo importante de recursos, puesto que es necesario no solo actualizar el software sino definir nuevos procesos de explotación, tarea siempre compleja y controvertida [25].

En contraposición, el Modelo SaaS, como modelo de negocio aplicado vía web, se encuentra almacenado en la nube (Cloud). La computación en la nube - Cloud Computing, ofrece un conjunto de recursos de computación en línea (procesamiento, almacenamiento, redes), que se pueden usar para ejecutar aplicaciones. Este cambio de modelo de negocio se puede ver reflejado en la Figura 1 y 2: 


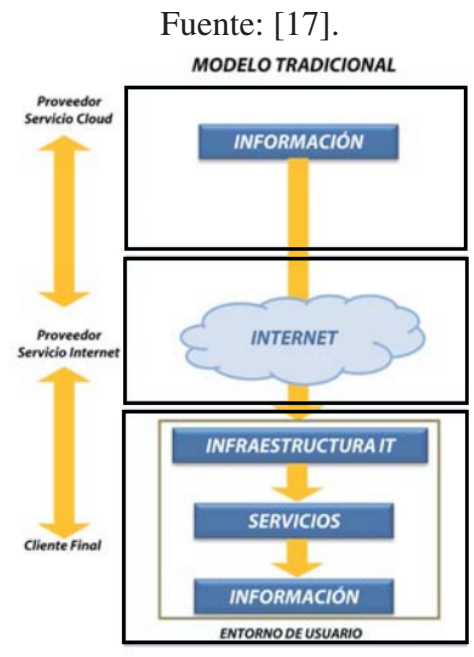

Figura 1. Modelo Tradicional.

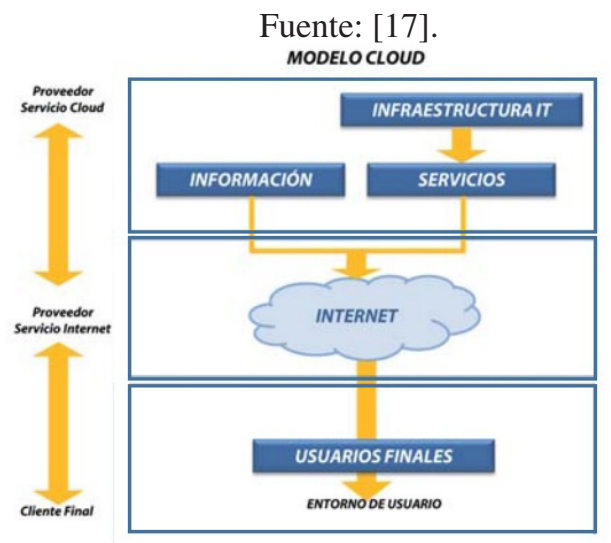

Figura 2. Modelo Cloud.

\section{DESARROLLO DE APLICACIONES CLOUD}

Para contextualizar este tema, en primera instancia es necesario esbozar el concepto de Cloud Computing.

\section{Qué es Cloud Computing?}

Según [19], Cloud Computing se define como un modelo que permite acceder a un conjunto compartido de recursos de cómputo, por ejemplo redes, servidores, almacenamiento y servicios; configurables bajo el esquema de demanda (Figura $3)$. Dichos recursos pueden ser rápidamente provistos y liberados con un mínimo esfuerzo administrativo o interacción con los proveedores de servicio.
En cuanto a cómo las empresas utilizan y adquieren los recursos de TI, Cloud Computing tiene como principal característica: la transformación de los modos tradicionales en un modelo de aprovisionamiento de recursos TI. Esta transformación potencia la prestación de servicios TI y servicios de negocio, facilitando la operatividad del usuario final y del prestador del servicio [13].

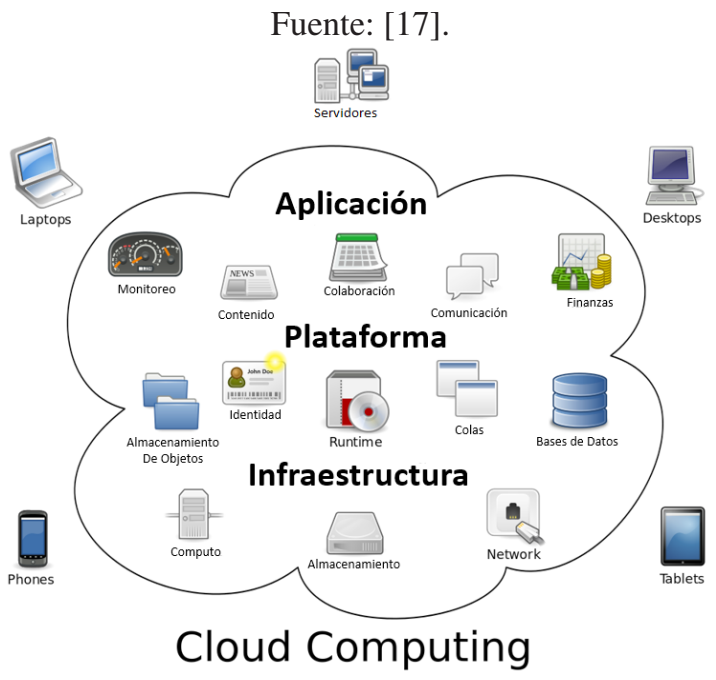

Figura 3. Servicios Cloud.

Este modelo de aprovisionamiento ofrece, entre otras características [26, 14]:

- Capacidad prácticamente ilimitada de procesamiento y almacenamiento.

- Pooling de recursos.

- Elasticidad, entendida como la capacidad de ampliar o reducir el uso de los recursos, con facilidad.

- Aprovisionamiento automatizado de servicios por demanda.

- Gran nivel de automatización.

- Facturación basada en el consumo.

Ahora bien, frecuentemente este tipo de modelo tiende a confundirse con otros existentes. Para evitar ambigüedades, a continuación se plantean diferencias que distinguen otros modelos del modelo de Cloud Computing, dentro de los cuales se tiene [3]:

1. Grid Computing: forma de computación distribuida, a través de la cual una 'súper computadora virtual', compuesta de un grupo 
de computadoras que se encuentran conectados a la red libremente, trabajan en conjunto para realizar tareas muy complejas.

2. Utility Computing: empaquetado de recursos computacionales, tales como capacidad de procesamiento y almacenamiento, medido de forma similar como los servicios tradicionales, ej.: servicio de electricidad.

3. Computación Autónoma: sistemas de computación capaces de auto-administrarse.

Aunque muchas implementaciones de Cloud Computing dependen de Grids de características autónomas, se enfoca más allá de dichas redes y de los servicios, haciendo uso de esquemas no centralizados, como por ejemplo las redes peer-to-peer como BitTorrent y Skype, entre otros. El modelo de acceso a los servicios y recursos usado por Cloud Computing se basa en cuatro estructuras de desarrollo principales [7]:

- Clouds privadas: son para el uso exclusivo de la organización, sean controladas o alojadas desde los Departamentos de TI de la empresa, o por parte de un proveedor externo.

- Clouds públicas: están abiertas a cualquier número de organizaciones y usuarios individuales de forma compartida. Si se utiliza una Cloud pública, se minimiza la inversión inicial y se combina agilidad y eficiencia con una capacidad de ampliación máxima.

- Clouds híbridas: combinan las Clouds privadas y públicas, de forma que ofrecen un acceso a recursos adicionales cuando la Cloud privada llega a su nivel de utilización máximo. Una Cloud híbrida podría dividir los recursos informáticos en niveles entre Clouds privadas y públicas. Por ejemplo, es posible que la base de datos resida en la Cloud privada, mientras que el servidor de aplicaciones lo haga en la Cloud pública.

- Clouds Comunitarias [14]: La infraestructura de la Cloud es provista para el uso exclusivo de una comunidad específica de consumidores que comparten los mismos objetivos (misionales, requerimientos de seguridad, políticas, consideraciones de cumplimiento). Estas pueden ser propiedad, administradas y operadas por una o más organizaciones de la comunidad, una tercera parte o una combinación de ellos.

Con cualquiera de estas estructuras, Cloud Computing permite que una aplicación aproveche la inactividad o el uso en exceso de la capacidad informática, de almacenamiento y de red, que se comparte con otras aplicaciones. Las soluciones basadas en Cloud constituyen una de las claves para evitar el exceso de aprovisionamiento, y para habilitar un equilibrio de carga eficiente entre recursos informáticos.

\section{Modelos de servicio en Cloud Computing}

Los fundamentos técnicos de las infraestructuras y los servicios de Cloud Computing incluyen la virtualización, software orientado a servicios, tecnologías de Grid Computing, gestión de grandes instalaciones y eficiencia de energía. Los consumidores compran este tipo de servicios en forma de "infraestructura como un servicio (IaaS)", "plataforma como servicio (PaaS)" o "software como un servicio (SaaS)" y venden servicios con valor agregado a los usuarios (Vakali, 2009). Como lo describen [14], y como se muestran en la Figura 4, existen tres modelos de servicio en Cloud Computing:

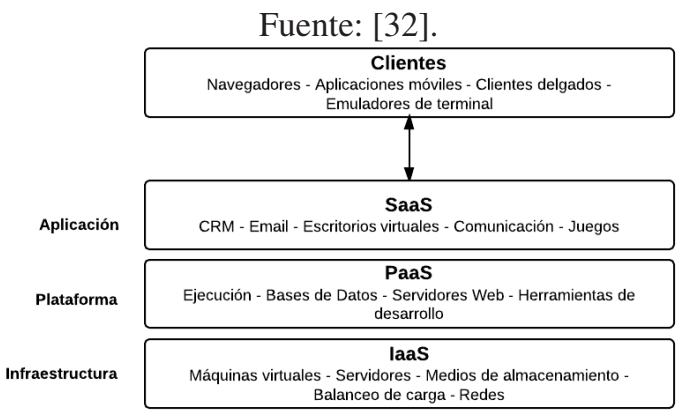

Figura 4. Modelos de Servicio Cloud.

- Software como Servicio (SaaS): aporta la capacidad de uso de aplicaciones del proveedor, que se ejecutan en una infraestructura Cloud, a través de un acceso web. Dichas aplicaciones son accesibles desde diferentes dispositivos cliente a través de una interfaz ligera o una interfaz de programa. El consumidor no administra ni controla la infraestructura Cloud, incluyendo la red, servidores, sistemas operativos, almacenamiento o incluso capacidades de aplicaciones individuales. Entre los ejemplos populares de SaaS se encuentran Google Docs, Salesforce, Dropbox, Gmail, Basecamp, etc.

- Plataforma como Servicio (PaaS): entorno de desarrollo y alojamiento de software compuesto por herramientas de programación, bases de datos, middleware y software de infraestructuras. 
El usuario despliega sus recursos en dicho entorno, utilizando lenguajes de programación, bibliotecas, servicios y herramientas provistas por el proveedor. El consumidor no administrar o controlar la infraestructura de Cloud incluyendo la red, servidores, sistemas operativos, o de almacenamiento, pero tiene control sobre las aplicaciones implementadas y posiblemente, los ajustes de configuración para el entorno de alojamiento de aplicaciones. Entre los ejemplos de PaaS se encuentran Google App Engine, que permite desarrollar aplicaciones en Java o Python desplegándolas en la infraestructura que provee Google.

- Infraestructura como Servicio (IaaS): habilita a los administradores del sistema y desarrolladores para lograr un aprovisionamiento automatizado de los recursos de computación, almacenamiento y red, que estos necesitan para desarrollar y ejecutar aplicaciones y sistemas operativos. El consumidor es capaz de desplegar y ejecutar software, que puede incluir sistemas operativos y aplicaciones. El consumidor no administrar o controlar la infraestructura de Cloud, pero tiene control sobre los sistemas operativos, almacenamiento y aplicaciones implementadas; y posiblemente un control limitado sobre la selección de los componentes de red (por ejemplo, firewalls de host). Entre los ejemplos populares de IaaS se encuentran Amazon Web Services EC2, GoGrid, etc.

Con independencia del modelo de servicio utilizado, el modelo de servicios integra diferentes niveles o capas de servicios TIC ofertados al cliente, como se muestra en la Figura 5 [17]:

a. Networking: red de interconexión como medio de comunicación entre los diferentes dispositivos que integran la infraestructura TIC.

b. Almacenamiento: capacidad de registro y de datos disponible en un determinado disco físico o virtual.

c. Servidores: equipamiento con capacidad para ejecutar determinadas aplicaciones. De esta manera el usuario final únicamente tendría que instalar las aplicaciones en el servidor, que estaría listo para ser utilizado en todo momento por cualquier usuario. d. Virtualización: capa de abstracción entre el hardware y el sistema operativo de la máquina virtual, haciendo posible la compartición de recursos entre diversos entornos de ejecución.

e. Sistema Operativo: programa o conjunto de programas que gestionan los recursos hardware (ya sean físicos o virtuales) disponibles, proveyendo acceso a las diferentes aplicaciones.

f. Middleware: capa de abstracción software (complejidad y heterogeneidad de las redes de comunicaciones subyacentes) que posibilita la comunicación entre las aplicaciones y los sistemas operativos o lenguajes de programación.

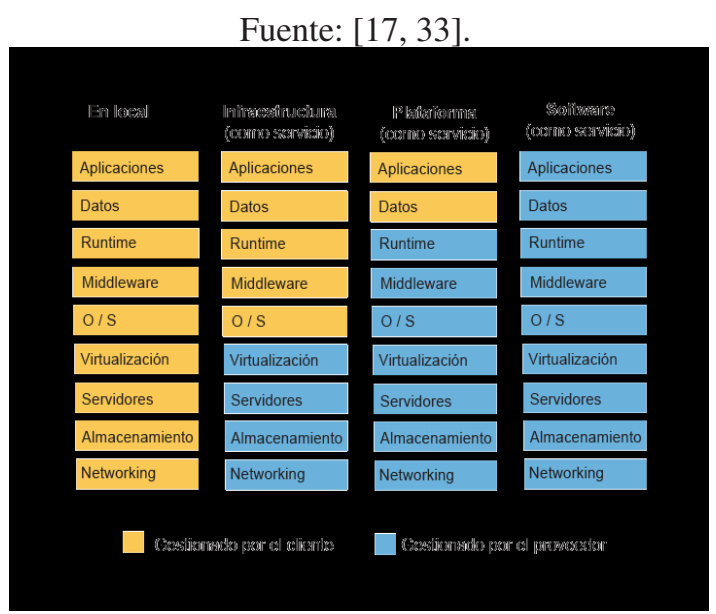

Figura 5. Modelos de Servicio.

\section{Ventajas y desventajas de Cloud Computing}

Al momento de decidir por una solución basada en Cloud, se debe analizar i) sus ventajas y desventajas, ii) el grado de incidencia de cada una de ellas en el core del negocio para el cual se busca su implementación, junto con iii) la pertinencia de la misma para la organización y el nicho de mercado. Posterior a ello, se deben explorar todas las expectativas que el mercado ofrece, evaluando cada uno de los aspectos requeridos, con el fin de tomar la mejor decisión al respecto. Para ello, es importante revisar algunas de las ventajas y desventajas que ofrece la Cloud Computing.

Como lo plantea [9], los beneficios que ofrece la Cloud son muchos, entre los cuales se describen:

- Escalabilidad: el precio para ampliar la plataforma (capacidad, potencia, etc.) es 
directamente proporcional a la ampliación que se necesite. Una empresa pequeña comprará una o dos licencias, mientras que una empresa mayor, que requiera más potencia, comprará 100, 40001000.

- Movilidad: Independencia del puesto físico de trabajo y de sus computadores. Al tener los datos en un servicio externo, cualquier computadora, cyber café o incluso Smartphone se convierten en una oficina, pudiendo acceder a los datos allá donde estés.

- Se ahorra en equipamiento, pues el cliente ya no se debe preocupar si sus equipos se quedan obsoletos, o si se han de cambiar, dado que la infraestructura corre a cuenta del proveedor. Es decir, se plantea una abstracción del soporte, mantenimiento e instalación.

- Se ahorra en eficiencia en caídas y backups, pues las empresas proveedoras se encuentran equipadas para hacer frente a posibles contingencias.

- Focalización [11]: Cloud Computing permite a las compañías centrarse en su core business. En vez de hacer una alta inversión tecnológica en sistemas, una empresa podría invertir en su infraestructura industrial, física o en capital humano, para proseguir sus planes de expansión

- Ecología [11]: Usar Cloud en una empresa reduce la huella de carbono de una empresa al ahorrar recursos y componentes, los cuales pasan de estar almacenados en componentes físicos a ser virtuales. Se ahorra también en consumo de energía con sus beneficios al medio ambiente.

- Disponibilidad del servicio o aplicación: 24 horas / 7 días / 365 días.

- Accesibilidad mediante diferentes tecnologías compatibles: como PDAs, móviles, portátiles, etc.

- Las actualizaciones de software son instantáneas [23]: el software en Cloud ya no se enfrentan a la elección entre software obsoleto y los altos costos de actualización. Cuando la aplicación está basada en la web, las actualizaciones desde el punto de vista del usuario, se hacen en forma automática y simplemente estarán disponibles la próxima vez que inicie su sesión en la Cloud.

- Disponibilidad de última versión [23]: En la Cloud siempre se aloja la última versión de sus documentos. Siempre y cuando se esté conectado, nunca estará en peligro de tener una versión antigua.

- Facilitar la colaboración en grupo [23]: Compartir documentos conduce directamente a la colaboración en documentos. Para muchos usuarios, esta es una de las ventajas más importantes de la computación en Cloud, dado que múltiples usuarios pueden colaborar fácilmente en documentos y proyectos.

De igual forma, [8] plantea 6 potenciales facilitadores que la Cloud empodera en la organización (Figura 6):

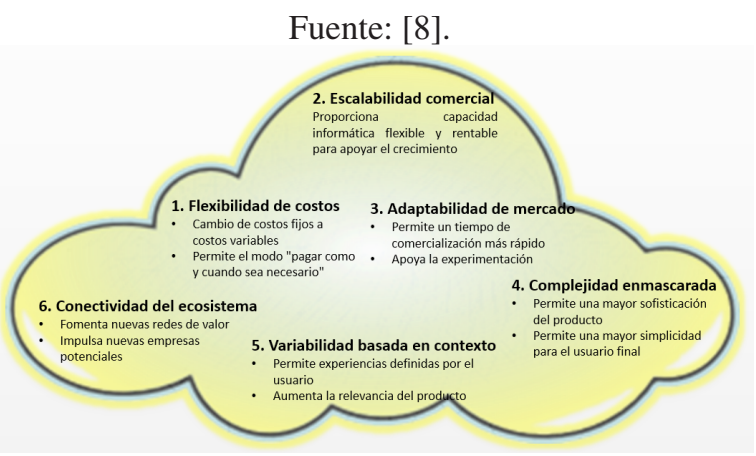

Figura 6. Facilitadores Organizacionales provistos por Cloud.

a. Flexibilidad de Costos: Cloud puede ayudar a una organización a reducir los costos de TI, al pasar de gastos de capital a gastos operativos. Hace uso del modelo "pay as and when needed", donde se paga por aquello que se consuma tal como CPU, megas consumidos, etc., o por su potencial consumo, como por ejemplo, el pago realizado por una cantidad de usuarios que usaran el hardware o software.

b. Escalabilidad del negocio: permite a la organización escalar fácilmente sus operaciones de negocio.

c. Adaptabilidad al mercado: adaptabilidad a las cambiantes necesidades del cliente, de tal forma que se pueda responder de una forma más rápida.

d. Complejidad enmascarada: ocultar a los usuarios lo intrincado de sus operaciones, lo que puede ayudar a atraer una gama más amplia de consumidores.

e. Variabilidad de Contexto: permite a las empresas ofrecer a los usuarios experiencias personales adaptadas a sus propios contextos, lo que permite una experiencia más centrada en el usuario. 
f. Conectividad de los Ecosistemas: facilita la colaboración externa con socios y clientes, lo que conlleva una mejora en la productividad y un aumento en la innovación.

Por otro lado, al momento de considerar las desventajas, tenga en cuenta que:

- Se depende completamente del proveedor y del buen uso de sus infraestructuras, así como de sus políticas de backups y actualizaciones.

- Conectividad: Sin acceso a Internet no hay Cloud Computing. Adicionalmente, este servicio puede caerse en cualquier momento por diversos factores.

- Mayor dependencia de la velocidad del servicio. Una conexión a Internet de baja velocidad hace que la computación en Cloud sea en muchos casos imposible. Las aplicaciones basadas en Web requieren una gran cantidad de ancho de banda para descargarse, al igual que documentos de gran tamaño [23].

- $\quad$ Seguridad [11]: Se debe ser muy cuidadoso con el manejo de la información para evitar que los datos sean robados por hackers o extraviados en agujeros de seguridad.

- Privacidad [11]: Datos confidenciales y sensibles como planes de mercadeo, lanzamientos de productos, información personal de empleados, datos financieros pueden quedar en manos de terceros si no se tienen las medidas preventivas.

\section{Tendencias de Cloud Computing}

Como lo plantea [21], el concepto de Cloud híbrido está tomando cada vez más fuerza, básicamente debido a que las grandes corporaciones con data centers in-house están buscando aprovechar las ventajas de la Cloud, integrando infraestructura propia y Clouds públicos a través de la tecnología existente. Basado en lo anterior, está comenzando a tomar forma una nueva estrategia entre algunos proveedores de servicios gestionados: el modelo Cloud Federation o federación de Clouds, como se muestra en la Figura 7.

Esta alternativa consiste en utilizar un software de orquestación mediante el cual se construyen y se gestionan recursos de diferentes Clouds públicas, aprovechando el potencial de todas ellas. Según [24, 35], plantea otras tendencias como:

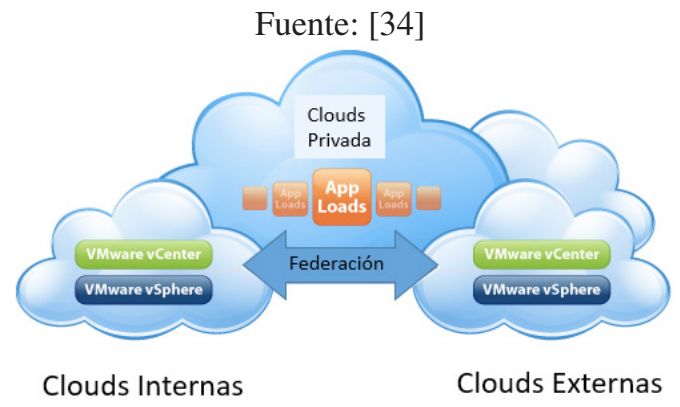

Figura 7. Cloud Federation.

a. Cloud es negocio: La Cloud es reconocida gracias a que facilita la "velocidad de salida al mercado",y permite conducir con agilidad el negocio. Esto se debe a que la Cloud apoya una rápida experimentación e innovación, facilitando que las empresas reaccionen de forma rápida e incluso adoptando nuevas soluciones sin costos iniciales significativos.

b. Seguridad: Abordar la seguridad y la privacidad es clave para generar confianza en Cloud Computing.

c. Cloud como una plataforma de innovación Móvil, Social (redes personales y de negocios), Big Data.

d. Social: colaboración en un contexto de negocios.

e. Big Data: Hasta nueve veces más de la información digital compartida en los últimos cinco años debe ser analizada y procesada. Big Data y el Análisis Predictivo se fusionarán y probablemente caerán bajo el concepto único de tecnología predictiva.

f. Realtime y Realtime Predictivo ya no es suficiente. Los usuarios son bombardeados con análisis y estadísticas en tiempo real. Pero en tiempo real ya no es suficiente. También tiene que ser predictivo.

g. Redes: El efecto de la red de negocios. Soluciones diseñadas para la economía en red se pueden aprovechar para asegurar la interacción entre todas las partes interesadas: empleados, socios, proveedores.

h. La Cloud Hibrida: La adopción de Cloud debe pasar en la comodidad del cliente y del negocio.

\section{SOFTWARE AS A SERVICE}

Como se describe en [1,27], Software como Servicio - SaaS, corresponde a un modelo de distribución de software mediante el cual una aplicación es ofrecida a múltiples clientes y es accesible a través de la red. Las principales características del modelo son:

a. El software es accesible, manejado y comercializado vía red.

b. El mantenimiento y actividades relacionadas con el software se realiza desde un lugar centralizado, 
en lugar de hacerlo en cada cliente, permitiendo a estos acceder a las aplicaciones vía la red.

c. La aplicación es distribuida, típicamente bajo el modelo de uno-a-muchos, incluyendo su arquitectura, management, precio y partnering.

d. Generalmente se basa en un modelo de comercialización en el cual no hay un costo inicial, sino que un pago por suscripción o por utilización en el cual no se diferencia la licencia del software del alojamiento del mismo.

Por otro lado, y de cara a una organización, como lo plantea [17], la principal ventaja asociada a la contratación SaaS a través de la nube, se sustenta sobre la no necesidad de un área específica de TI que se encargue de la gestión del software. Bajo este paradigma, será el proveedor de servicios Cloud, quien se encargue de asegurar al cliente la seguridad de acceso a las aplicaciones, su disponibilidad y el correcto funcionamiento. Además, la gestión de licencias ya no es necesaria, y el sistema operativo sobre el que corren las aplicaciones puede ser seleccionado por el cliente en función de sus necesidades y/o preferencias. Dentro de SaaS, existen dos tipologías claramente diferenciadas:

a. Business Applications: aplicaciones cuyo principal objetivo es ayudar a las organizaciones a llevar a cabo sus tareas de manera rápida y precisa.

b. Development Tools: herramientas que se utilizan en las organizaciones para el desarrollo y la administración de sus productos.

\section{La Arquitectura SaaS}

$\mathrm{La}$ arquitectura SaaS puede clasificarse dentro en cuatro "niveles de madurez", donde los atributos principales son fácil configuración, eficiencia multitenant y escalabilidad. Cada nivel se distingue del anterior por la adición de uno de estos atributos, los cuales se describen a continuación [4]:

- Nivel 1 -Ad-Hoc / personalizado: cada cliente cuenta con su propia versión de la aplicación, hospedada y ejecuta su propia instancia de la aplicación en los servidores de hospedaje.

- Nivel 2 - Fácil configuración: proporciona mayor flexibilidad de programación a través de meta-datos configurables, de manera que varios clientes puedan utilizar instancias separadas del mismo código de la aplicación. Permitiendo al proveedor satisfacer diferentes necesidades a través de opciones de configuración detalladas, simplificándose el mantenimiento y la actualización del código base, el mismo que es común a todos los clientes.

- Nivel 3-Configurable y eficiencia Multi-Tenant: agrega el concepto de "multi-tenancy" al segundo nivel, de manera que una sola instancia del programa se ejecuta para todos los clientes. Esto hace que el uso de recursos del servidor sea más eficiente, de tal forma que es completamente transparente para el usuario final. Sin embargo, y en el transcurso del tiempo, se tropieza con los límites de escalabilidad.

- $\quad$ Nivel 4 - Escalable, configurable y eficiencia Multi-Tenant: agrega la escalabilidad a través de una arquitectura que soporta un "conjunto" de instancias de aplicaciones ejecutándose en varios servidores, haciendo uso de un balanceo de carga. El proveedor puede incrementar o disminuir la capacidad del sistema, a fin de cumplir con la demanda, adicionando o retirando servidores, sin la necesidad de alterar o modificar la arquitectura de las aplicaciones.

\section{Requisitos Mínimos que debe Ofrecer SaaS}

Como lo expone [2], desde el punto de vista del cliente que va a adquirir los servicios de una aplicación ofrecida como servicio, existen una serie de requisitos mínimos necesarios que un SaaS debe ofrecer:

a) Rendimiento. Un SaaS debe ofrecer un rendimiento mínimo y aceptable para que sea atractiva su adquisición. El problema aquí es definir cuál es el rendimiento mínimo y aceptable. Aunque es un concepto subjetivo, puede ser medible en tiempos de respuesta en el acceso a los datos, de ejecución los procesos de negocio, de comunicación a la propia aplicación, etc.

b) Acuerdo de Nivel de Servicio (Service Level Agreement). El proveedor de la aplicación SaaS debe proveer varios niveles de servicio al que los clientes puedan adherirse. Habrá clientes que necesiten su aplicación disponible $8 \times 5$ ( 5 días a la semana, $8 \mathrm{~h}$ ), y habrá que clientes que necesiten 24 X 7. El proveedor deberá instalar en sus sistemas, los mecanismos necesarios para poder ofrecer este tipo de acuerdos, como por ejemplo backup, cluster de alta disponibilidad de datos y aplicación, etc. 
c) Privacidad en las comunicaciones. Debido a la importancia de los datos que puedan albergar las aplicaciones, es necesario que la comunicación que se realiza a través de Internet sea segura. Dicha comunicación debe realizarse a través de https, u otra forma de comunicación que asegure la privacidad de las comunicaciones.

d) Privacidad de los datos. El proveedor debe asegurar que los datos estén seguros y accesibles única y exclusivamente por el dueño del dato. Esto debe ser especialmente perseguido en la aplicaciones multitenant (nivel 3 y 4 de madurez).

e) Monitoreo de la aplicación. El cliente debe saber, de alguna forma, que es lo que ocurre en su aplicación, por ejemplo: quién accede, a qué procesos, a qué datos, etc. Esto es necesario cuando el pago por el uso de la aplicación se realiza a través de conceptos como horas de utilización de la aplicación, consumo de espacio de disco, o cualquier otra forma que sea variable.

f) Acceso a los datos. El resto de la aplicaciones de la organización deben acceder a través de APIs o de Web Services, a los datos y lógica de negocio que se utilizan y generan por el uso de la SaaS, sobretodo en clientes que tengan adoptado la arquitectura SOA en su sistema de información.

\section{Tipos de Servicios SaaS}

Dentro de los servicios ofertados en plataformas SaaS (como los descritos en sitios web como http:// www.portalsaas.com/) se encuentran:

a. Aplicaciones web, tales como CMS o gestores de contenidos, CRMs, WebOS (Webdesktops o escritorios basados en web), correo, mensajería instantánea, plataforma educativa, comercio electrónico, sistemas de pago, herramientas colaborativas, etc.

b. Otras aplicaciones tales como acceso a bases de datos, aplicaciones mixtas, etc.

c. Almacenamiento Remoto y backup.

d. Control Remoto para soporte y formación.

e. Alquiler de la plataforma para servicios/aplicaciones de terceros o soluciones tradicionales de hosting.

f. Repositorio de software

g. Servicios Web

\section{LEARNING MANAGEMENT SYSTEM - LMS}

Como lo expone [5], una Plataforma de Gestión del Aprendizaje es un software que automatiza la administración de los eventos de capacitación. El LMS permite la inscripción de los usuarios a los distintos programas formativos, lleva el registro de desempeño de los alumnos y proporciona informes para la gestión de la organización. Un LMS está diseñado típicamente para editores y proveedores múltiples, permitiendo administrar cursos desarrollados a partir de diversas fuentes, o bien incluir capacidades de creación de contenidos (LCMS). Los LMS, aportan importantes herramientas al proceso educativo (Figura 8), generalmente [20]:

- Herramientas de gestión y distribución de contenidos. Permiten almacenar, organizar, recuperar y distribuir contenidos educativos y estructurarlos en contenidos de mayor complejidad y alcance temático.

- Herramientas de administración de usuarios. Facilitan el registro de los usuarios del sistema para el posterior control de acceso y presentación personalizada de los contenidos y cursos.

- Herramientas de comunicación. Chats, foros, correo electrónico, tableros de anuncios, permiten la comunicación entre estudiantes y tutores en una vía o en doble vía, sincrónica y asincrónicamente.

- Herramientas de evaluación y seguimiento. Apoyan la construcción y presentación de evaluaciones mediante la utilización de diferentes tipos de preguntas: abierta, falso o verdadero, selección múltiple, múltiple opción, completar y apareamiento entre otras. Algunas veces también permite la construcción de bancos de preguntas usados con frecuencia para seleccionar aleatoriamente preguntas para los estudiantes.

Fuente: [16].

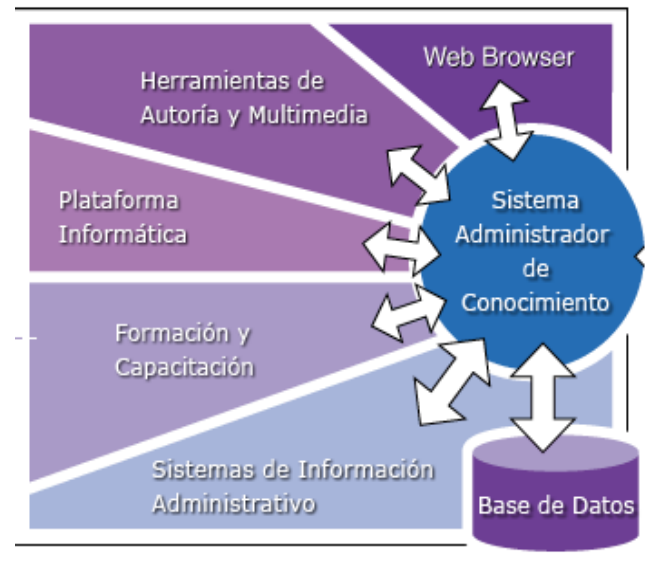

Figura 8. Herramientas LMS. 
En términos generales, un LMS gestiona a) el seguimiento y reporte de las actividades de aprendizaje individual y grupal; b) el sistema de creación de contenido (LCMS) permite que la unidad de formación cree y/o suba los contenidos o cursos creados in-house o comprados, y c) contenidos o cursos propios [18]. Con el fin de elegir un LMS adecuado, es primordial identificar las necesidades del negocio, dado que procesos como las adaptaciones pueden llegar a incluir costos adicionales a la adquisición del LMS. De igual manera, es importante revisar las funcionalidades que ofrece la herramienta, tales como crear o cargar cursos, crear contenido, sistema de registro, catálogo de cursos, bibliotecas digitales, seguimiento del desempeño de los estudiantes, mecanismos de autoevaluación, estadísticas e información de cursos y estudiantes, apoyo a comunidades de aprendizaje, etc.

\section{TIPOS DE LMS}

Para llevar a cabo la elección del LMS a usar, es importante tener en cuenta las características bajo las cuales se planea llevar a cabo la formación de la población objeto. Dadas estas características, se pueden contemplar diferentes tipos de LMS, dentro de los cuales se identifican [15]:

\section{LMS Libre vs Comercial}

Una de las primeras preguntas que se debe cuestionar al momento de seleccionar un LMS es: Libre, Código Abierto? Comercial?

Un LMS Libre, regularmente no tiene costo, y dada su característica de ser código abierto, éste es posible de ser adaptado a las características y requerimientos particulares de la organización. Ante esta decisión es primordial tener en cuenta que adaptar cualquier herramienta requiere de un proceso algunas veces muy técnico. Por otro lado, en un porcentaje de estos productos, el soporte encontrado es restringido o en algunos casos casi nulos, limitándose muchas veces a foros o a sitios en la red, donde los usuarios enlazan sus preguntas en busca de alguna colaboración de la comunidad virtual.

Dentro del catálogo de LMS Open Source se encuentra algunos como:

- eFront.

- Dokeos.
- Claroline.

- ATutor.

- ILIAS.

- OpenOLAT.

- Sakai.

- LRN.

- Ganesha.

- Latitud eLearning.

- Chamilo LMS.

- Moodle.

Es importante destacar que algunos de ellos ofrecen más o menos funcionalidades, soporte, estabilidad, etc.

En cuanto a un $\mathbf{L M S}$ propietario, que por ende tiene un costo comercial, están apoyados por una estrategia de soporte, sea presencial, en línea, telefónica, etc. Es importante tener en cuenta que dado su carácter propietario, las parametrizaciones las realiza el proveedor, acorde a las solicitudes de los clientes. De igual forma, dependiendo del tipo de contrato de licenciamiento, las actualizaciones pueden descargarse gratis (en un periodo de tiempo), o en su defecto tiene un costo comercial adicional. Dentro de los LMS propietarios, se pueden identificar aquellos que ofrecen una licencia para ser instalado localmente o aquellos que son ofrecidos como servicios desde la nube. La Tabla 1 muestra algunos ejemplos del catálogo de LMS Propietario.

\section{LMS Instalado vs SaaS.}

Cuando se adquiere un producto de software, sea cual sea este, se deben tener en cuenta diferentes aspectos positivos o negativos (algunos generados por el imaginario colectivo), acerca de la forma como se adquiere dicho producto, y para este caso de estudio, un LMS.

Un producto de software adquirido para ser instalado localmente, debe contar con personal de TI para administrar, dar soporte, personalizar y escalar dicho producto. Además, se debe contar con la infraestructura necesaria para mantener, ofertar las funcionalidades del producto de software, en este caso del LMS; etc.

Por otro lado, los productos SaaS basados en la nube, son más flexibles de escalar. Debido a que el producto se vende como un "servicio", mucho de los requerimientos tecnológicos, operativos y administrativos son pasados al proveedor. 
Específicamente para un LMS, una de las principales características a tener en cuenta es la capacidad de escalar de forma rápida y sencilla. Muchos LMS comerciales son vendidos como productos locales o como SaaS. La Tabla 1 muestra algunos LMS SaaS que se encuentran en el mercado.

Tabla 1. Ejemplos de LMS Propietarios y SaaS.

\begin{tabular}{|l|l|}
\hline \multicolumn{1}{|c|}{ LMS Propietario } & \multicolumn{1}{c|}{ LMS SaaS } \\
\hline EdModo. & Docebo. \\
sumTotal. & BlackBoard. \\
eDucativa. & TalenTLms. \\
Schoology. & MindFlash. \\
BlackBoard. & Litmos. \\
iLearning by Oracle. & Inquisiq ${ }^{\text {r3. }}$. \\
Aulapp. & Haiku. \\
Desire2Learn. & Integrated LMS. \\
eCollege. & Skill Soft. \\
Fronter. & ScolarLms. \\
Saba Learning. & ELearning247. \\
& Expertus. \\
& OpenClass. \\
& MoodleRooms. \\
& Risc. \\
& Learning Evolution. \\
\hline
\end{tabular}

Fuente: Autores.

\section{Creación de Cursos o No?}

Adicionalmente, se debe analizar si el LMS viene con la funcionalidad de crear contenidos de formación. En algunos casos, un LMS es simplemente una herramienta para distribuir el contenido del curso, que debe ser creado en un Sistema de Gestión de Contenidos de Aprendizaje (LCMS).

Algunos sistemas LMS, sin embargo, proveen herramientas de autor que permiten a los formadores y diseñadores, desarrollar su propio contenido único. Otros proveedores LMS ofrecen herramientas para la creación de cursos, de forma separada, y otros ofrecen materiales de curso creados por terceros. En cualquiera de los casos anteriores, es importante verificar que la creación de cursos este cobijada o sea compatible por la aplicación de algún estándar como por ejemplo SCORM.

\section{Integración con Otras Aplicaciones}

Por último, se debe verificar que el LMS ofrezca estrategias de integración con otras aplicaciones que posee o contrata la organización, tales como calendarios, correo electrónico o redes sociales como Facebook y Twitter, sistemas de gestión internos / externos (contratación, desempeño, nomina, etc.). En términos generales, la definición de una arquitectura de interoperabilidad que posibilite la integración de aplicaciones en los LMS y la exportación de su información a otros contextos.

\section{INTEROPERABILIDAD DE APLICACIONES Y LMS}

Además de poseer determinadas estrategias de seguridad, soporte, infraestructura, servicios, etc.; según el tipo de LMS que se maneje, es de vital importancia poder articularlo con las demás aplicaciones que la organización use en el proceso de aprendizaje. Por ejemplo, para el caso de una Universidad, sería importante poder articular el LMS con: i) el sistema de registro de estudiante, de tal forma que los estudiantes matriculados puedan tener acceso al LMS; ii) registrar el desempeño académico de los estudiantes, iii) evaluar los materiales docentes, iv) activar o desactivar docentes, v) gestionar los componentes "semilla" de los espacios académicos (objetos de aprendizaje, etc.), entre otras actividades.

En muchas instituciones no hay ningún proceso de integración, de tal forma que las aplicaciones funcionan de forma independiente Por ejemplo, el docente / tutor registra el seguimiento de los estudiantes en el LMS, y posteriormente tiene que ingresar y diligenciar el sistema de registro de notas de la institución.

Por otro lado, otras instituciones han incursionado en el uso de mecanismos como SnapShots, que permite mover grandes volúmenes de datos, permitiendo a los sistemas integrados poder solicitar, generar y cargar archivos. Por ejemplo, un sistema de registro, como un sistema de información de estudiantes, puede generar un snapshot y enviar la notificación a un sistema consumidor, como un LMS. Dicho sistema consumidor es notificado de la ubicación del archivo, con lo cual puede iniciar su consumo.

Un ejemplo de la necesidad de este proceso de integración de aplicaciones es el expuesto por [6], donde se muestra como la Universidad Autónoma de Madrid dispone de la aplicación para gestión académica Sigma, que gestiona todo el ciclo de vida de un estudiante, y por otro lado, la herramienta corporativa Moodle, seleccionada por la UAM para impartir docencia en red.

Estos dos sistemas de información han llevado vidas paralelas, de modo que desde Moodle no era posible 
aprovechar la información contenida en el sistema de gestión académica de un modo eficiente. Por ello, los profesores Moodle debían crear sus propios cursos, asignar a los alumnos a los mismos, etc., de modo absolutamente manual. Asimismo, los datos recogidos en Moodle en cuanto a evaluaciones se refiere, no podían ser transmitidos a la aplicación de gestión académica de un modo automático; el profesor debía realizar esa tarea manualmente. Esta situación planteó un reto de integración para la UAM logrando un proceso de integración del Campus Virtual (plataforma de e-learning Moodle) con la aplicación institucional de gestión académica, como se muestra en la Figura 9.

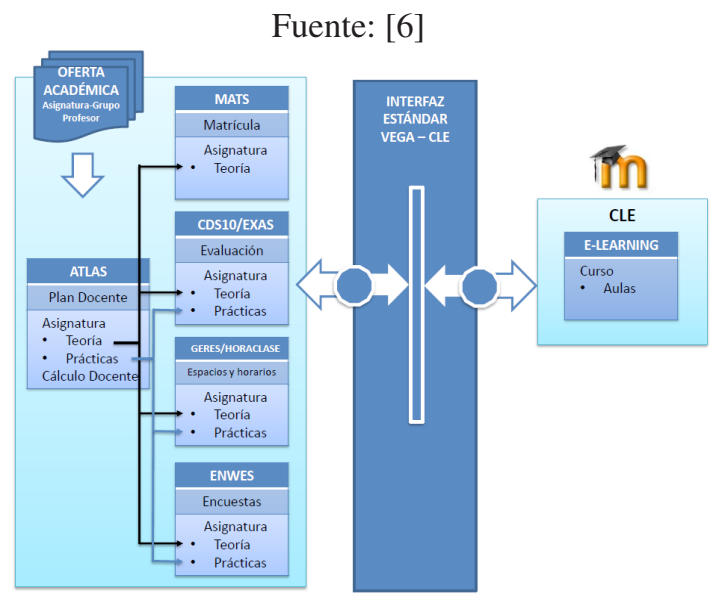

Figura 9. Integración Campus Virtual UAM.

En términos generales, estos procesos de integración deben regirse por estándares como el LTI - Learning Tools Interoperability [12], gestionado por el IMS Global Learning Consortium [10]; uno de los estándares más usados puesto que permite integrar aplicaciones y contenidos en entornos virtuales de aprendizaje (LMS) de forma rápida y segura.

La función principal de LTI es establecer un procedimiento estándar para integrar aplicaciones de aprendizaje (a menudo alojados de forma remota y proporcionada a través de los servicios de terceros), con plataformas como los LMS, portales, u otros entornos educativos. En LTI estas aplicaciones de aprendizaje son llamadas Herramientas (proporcionadas por los proveedores de herramientas), y el LMS u otras plataformas, denominadas Consumidores de Herramientas.

El caso de uso básico detrás del desarrollo de la especificación LTI es permitir la conexión, sin problemas, de aplicaciones y contenidos basadas en web, alojados externamente, o herramientas, a las plataformas que les presentan a los usuarios [12].

Fuente: [12].

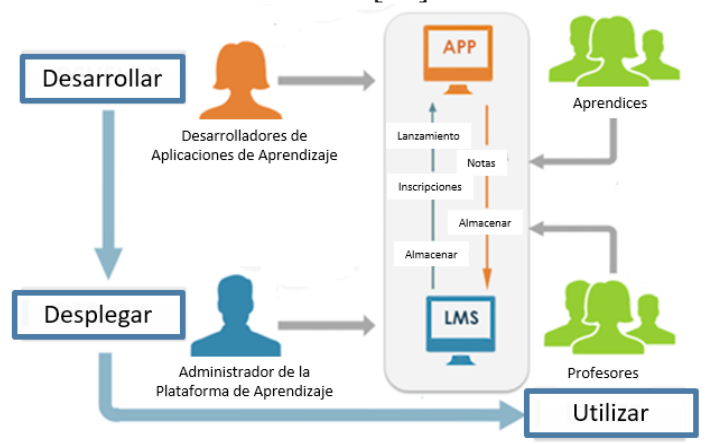

Figura 10. LTI - Learning Tools Interoperability.

Como se muestra en la Figura 10, la aplicación de la especificación LTI busca que, por ejemplo:

- Los Estudiantes o Aprendices, no tengan que registrarse de forma separada en diferentes aplicaciones para poder lograr la completitud del proceso de aprendizaje;

- Los profesores puedan vincular diferentes aplicaciones y tener acceso instantáneo;

- Los administradores de ambientes virtuales de aprendizaje (VLE) puedan actualizar sus sistemas y adicionar nuevas aplicaciones, etc.

\section{¿Cuál LMS SaaS escoger?}

En síntesis, acorde a las necesidades de la institución, se pueden establecer diferentes criterios para la selección del LMS SaaS, tales como:

- Arquitectura: Servicios Cloud Nativos, gestión de carga automatizada, tecnologías de desarrollo, etc.

- Apertura: Licenciamiento, API, auditorias de seguridad, etc.

- Características: funcionalidades, integración de aplicaciones, aplicaciones móviles, etc.

- Usabilidad: accesibilidad.

Entre otros factores que permitirán tomar la decisión acerca de cuál plataforma elegir, disponible en el mercado, tales como:

- Open Source: Chamilo, Moodle, etc.

- SaaS: Canvas, E-doceo, etc. 
Para revisar la comparación de características entre LMS, puedes visitar portales como [28-30]. Muchas de estas características se comparan en relación con los comentarios de los usuarios, como por ejemplo en [31], donde Canvas tiene 4.3/5 estrellas con 278 comentarios, y Moodle obtiene 3.8/5 estrellas con 141 comentarios.

\section{TRABAJOS FUTUROS}

En primera instancia, se plantea como trabajos futuros la finalización del presente proyecto de investigación que servirá de complemento a diferentes instituciones que inicien el proceso de inserción en los escenarios de e-learning y virtualización de contenidos. Como segunda instancia, se proyecta el diseño de una propuesta para una línea de producción tecnológica de Objetos de Aprendizaje para la Universidad Distrital Francisco José de Caldas, en términos de workflow, infraestructura, hardware y software base requeridos para tal fin; dado que dicha institución se encuentra en la actualidad en la etapa de operacionalización de conocimiento acerca del tema, e ingresando a la oferta educativa virtual.

En tercera instancia, este proyecto de investigación contribuirá al fortalecimiento de la investigación en e-learning y virtualización, al interior del grupo de Investigación GIIRA, de la Facultad de Ingeniería de la Universidad Distrital Francisco José de Caldas, y de igual forma, ofertar nuevos escenarios de investigación para los estudiantes de pregrado y posgrado de dicha institución.

En cuarta instancia, como recomendaciones del proyecto de investigación en el cual se enmarca el presente artículo, se plantea la exploración de los servicios SaaS y su integración con las demás aplicaciones, como estrategia para disminuir los costos de inversión de la inmersión en los procesos de formación virtual, dado las limitantes presupuestales y técnicas que puedan surgir para ampliar la infraestructura física y tecnológica requerida para dar soporte a este proceso de enseñanza aprendizaje.

\section{CONCLUSIONES}

Los SaaS LMS se convierten en una estrategia llamativa, al momento de incorporar el e-learning a los procesos de formación. La selección de estas herramientas deben responder a un proceso planificado y metódicamente guiado, donde se analicen aspectos como el core del negocio, la población objeto, los tipos de formación realizada, los requerimientos organizacionales y demás aspectos de la gestión de la configuración con los que cuenta y debería contar la organización que decida implementarlos.

Como lo plantea para obtener un máximo de aprovechamiento de un modelo de servicio como SaaS, como estrategia en la organización, se deben tener en cuenta factores como a) Explotar la adopción de SaaS a nivel "general", en paralelo con un enfoque estratégico, para definir cómo SaaS puede impulsar el valor empresarial sostenible; b) Identificar los cambios de comportamiento clave contra el mapa de procesos de negocio, utilizando métricas SaaS para promover y realizar el seguimiento del cambio; c) Desarrollar un plan de arquitectura para guiar la integración y gestión de datos maestros para minimizar el reproceso en un ciclo iterativo; d) Sentar las bases para la nueva colaboración permanente entre el negocio y TI. No hay que subestimar el impacto de las diferencias organizacionales en el soporte del modelo SaaS; y e) Crear un ambiente de aprendizaje donde $\mathrm{SaaS}$ permite conectar rápidamente la corrección del curso y la transformación gradual.

En cuanto a la articulación de los SaaS LMS a los procesos de aprendizaje, las plataformas SaaS ofrecen una experiencia de calidad para el alumno, mientras le ahorro de dinero a la organización a través de la eliminación de gastos en la construcción de un LMS in-house, lo cual redundaría en tener más recursos para invertir directamente en la formación de las personas. De igual forma, los SaaS LMS eliminan el costo de hardware de servidor, dispositivos de almacenamiento y equipos de red, actualizaciones del sistema operativo, los derechos de licencia de software, etc. Y demás recursos técnicos, operativos y administrativos necesarios para su operación. Mediante el outsourcing de sus necesidades de LMS, una empresa puede ahorrar valioso tiempo y dinero [22].

Adicionalmente, es necesario analizar la integración de los diferentes sistemas de gestión académica con los SaaS LMS, de tal forma que se ofrezca a la comunidad una alternativa de solución integral, que le permita realizar una gestión eficiente, en lugar de 
adicionar tareas o duplicar procesos, por no contar con una adecuada estrategia de interoperabilidad entre las aplicaciones.

\section{AGRADECIMIENTOS}

Los autores expresan sus agradecimientos al Grupo de Investigación GIIRA y a la Universidad Distrital Francisco José de Caldas, Grupo y Universidad en los cuales se lleva a cabo este proyecto.

\section{REFERENCIAS}

[1] Santos, M. SaaS, IaaS y PaaS: i Qué son, Cómo usarlos y Para qué? Enter. co. 2015. Fecha de consulta: 17 de febrero de 2017. URL: https://www.enter.co/guias/ tecnoguias-para-empresas/saas-iaas-y-paasque-son-como-usarlos-y-para-que/

[2] J. Arévalo Navarro. "Cloud Computing: fundamentos, diseño y arquitectura aplicados a un caso de estudio". Tesis de Fin de Máster. Máster Oficial en Tecnologías de la Información y Sistemas Informáticos. Universidad Rey Juan Carlos. 2011. Fecha de consulta: 10 de Abril de 2015. URL: http:// eciencia.urjc.es/bitstream/10115/5945/1/ MemoriaTFMFINAL-2.pdf

[3] CCA. "Qué es Cloud Computing". Cloud Computing América. 2018. Fecha de consulta: 01 de marzo de 2018. URL: https://www.cloudamerica.com/que-es-cloud-computing.html

[4] CCA. "Qué es SaaS". Cloud Computing América. 2018. Fecha de consulta: 1 de Marzo de 2018. URL: https://www.cloud-america. com/que-es-saas.html

[5] Creatic. "El top 5 de las mejores plataformas de E-Learning". 2017. Fecha de consulta: 1 de Abril de 2017. URL: https://creatic.co/el-top5-de-las-mejores-plataformas-de-e-learning/

[6] Cuadrón, O. Gómez y M. "Docencia en Red: integración de la gestión académica y las herramientas docentes. SIGMA + Moodle". Universidad Autónoma de Madrid. 2013. Fecha de consulta: 13 de Abril de 2016. URL: https://www.uam.es/gruposinv/dim/assets/ melchor2013.pdf

[7] Dell. "En qué consiste Cloud Computing?". Dell Inc. 2018. Fecha de consulta: 2 de Marzo de 2018. URL: https://www.dell.com/learn/ es/es/esbsdt1/dell-cloud-computing.

[8] S. Berman, L. Kesterson-Townes, A. Marshall and R. Srivathsa. "The Power of Cloud: Driving business model innovation". IBM Global Business Services. 2012. Disponible en: http://www.ibm.com/cloud-computing/ us/en/assets/power-of-cloud-for-bus-modelinnovation.pdf. Date of visit: April 10, 2015.

[9] IDaccion. "Ventajas e inconvenientes del Cloud computing para tu empresa". IDaccion Business News. 2015. Fecha de consulta: 02 de Abril de 2016. URL: http://idnews. idaccion.com/ventajas-e-inconvenientesdel-cloud-computing-para-tu-empresa/

[10] IGLC. "IMS Global Learning Consortium". 2018. Fecha de consulta: 15 de Febrero de 2018. URL: http://www.imsglobal.org/

[11] Enae. "Ventajas y desventajas del Cloud Computing". 2010. Fecha de consullta: 08 de Abril de 2016. URL: http://www.enae.es/blog/ ventajas-y-desventajas-del-cloud-computing.

[12] IMS. "Learning Tools Interoperability". 2018. Fecha de consulta: 16 de Marzo de 2018. URL: http://www.imsglobal.org/ toolsinteroperability2.cfm

[13] M. Maruzzo, F. Millán, N. Rodríguez, D. Segura y D. Villafañe. "Desarrollo de Aplicaciones para Cloud Computing". Universidad Nacional de San Juan. CACIC 2010 - XVI Congreso Argentino de Ciencias de la Computación. 2010. Fecha de consulta: 28 de Marzo de 2016. URL: http://sedici.unlp.edu.ar/bitstream/ handle/10915/19374/Documento_completo. pdf?sequence $=1$

[14] P. Mell and T. Grance. "The NIST Definition of Cloud Computing. Recommendations of the National Institute of Standards and Technology". US Department of Commerce. National Institute of Standards and Technology. 2011. Fecha de consulta: 10 de Mayo de 2015. URL: http://nvlpubs.nist.gov/nistpubs/Legacy/ SP/nistspecialpublication800-145.pdf

[15] Mindflash. "Types of Learning Management Systems". MindFlash. 2018. Fecha de consulta: 30 de Marzo de 2018. URL: https://www. mindflash.com/learning-management-systems/ types-of-lms 
[16] M@@dle Chile. “Plataforma LMS”. 2016. Fecha de consulta: 19 de Abril de 2018. URL: http://www.moodle-chile.cl/

[17] Management Solutions. "La Nube: oportunidades y retos para los integrantes de la cadena de valor". 2012. Fecha de consulta: 11 de Abril de 2016. URL: http:// www.managementsolutions.com/PDF/ESP/ La-nube.pdf

[18] Nielsen, B. "Learning Management System Basics (LMS)". 2017. Fecha de consulta: 1 de Abril de 2017. URL: http://www.yourtrainingedge.com/ learning-management-system-basics-lms/

[19] NIST. "NIST Cloud Computing". National Institute of Standards and Technology. US Department of Commerce. 2017. Fecha de consulta: 9 de Enero de 2018. URL: http:// www.nist.gov/itl/cloud/

[20] L.Ortiz. "Campus Virtual: la educación más allá del LMS". Revista de Universidad y Sociedad del Conocimiento (RUSC). Vol. 4, $\mathrm{N}^{\circ}$ 1. UOC. ISSN 1698-580X. 2007. Fecha de consulta: 25 de Marzo de 2014. URL: https://www.researchgate.net/ publication/28161098_Campus_Virtual_ la_educacion_mas_alla_del_LMS

[21] A. Pérez. "Actores y Tendencias en la Nube. ¿Rumbo al Cloud Federation?”. Revista Cloud Computing. 2014. Fecha de consulta: 10 de Abril de 2016. URL: http:// www.revistacloudcomputing.com/2014/04/ actores-y-tendencias-en-la-nube-rumbo-alcloud-federation/\#comment-3445

[22] ProProf Training Marker. "10 reasons why a SaaS LMS is the most cost-effective choice". 2013. Fecha de consulta: 19 de Abril de 2016. URL: http://www.proprofs.com/c/ lms/10-reasons-why-a-saas-lms-is-the-mostcost-effective-choice/

[23] Prospecnet. "Computación en la Nube: Una nueva moda, un nuevo concepto o una nueva verdadera revolución en la forma de brindar servicios de TI?". 2015. Fecha de consulta: 15 de Marzo de 2017. URL: http://www. prospecnet.com/A_LaNube.aspx

[24] TeleCarrier. "Tendencias Cloud Computing 2018". 2017. Fecha de consulta: 11 de Febrero de 2017. URL: https://www.telecarrier.com/ blogs/tendencias-cloud-computing-2018
[25] S. Ros Muñoz. "Sistemas de E-learning abiertos basados en servicios". Tesis Doctoral. Escuela Técnica Superior de Ingenieros Industriales. Universidad Nacional de Educación a Distancia. Madrid. 2012. Fecha de consulta: 20 de Mayo de 2015. URL: http://e-spacio.uned.es/fez/eserv/ tesisuned:IngInd-Sros/Documento.pdf

[26] A. Vakali, P. Mehra, D. Katsaros, M. Dikaiakos and G. Pallis. "Cloud Computing: Distributed Internet Computing for IT and Scientific Research". IEEE INTERNET COMPUTING. 1089-7801. 2009. Fecha de consulta: 15 de Junio de 2015. URL: http://ieeexplore.ieee. org/document $/ 5233607 /$ ?reload=true

[27] M. Woloski. "Aplicaciones de Software como Servicio". Tesis de Grado. Facultad de Ingeniería. Universidad de Buenos Aires. 2008. Fecha de consulta: 20 de Marzo de 2014. URL: http://materias.fi.uba.ar/7500/ woloski-tesisingenieriainformatica.pdf

[28] J. Polo. "Canvas, una nueva plataforma para crear y dar cursos. Tecnología, Internet y Marketing”. 2012. Fecha de consulta: 9 de Enero de 2018. URL: https://wwwhatsnew. com/2012/10/22/canvas-una-nuevaplataforma-para-crear-y-dar-cursos/

[29] FinancesOnline. "Compare Moodle vs. Canvas LMS”. 2017. FinancesOnline. Fecha de consulta: 9 de Enero de 2018. URL: https://comparisons.financesonline.com/ canvas-lms-vs-moodle

[30] M. Pardo. "Moodle vs. Canvas: Key Features and Services Comparison". 2017. BetterBuys. Fecha de consulta: 9 de Enero de 2018. URL: https://www.betterbuys.com/lms/ moodle-vs-canvas/

[31] G2Crowd. "Compare Canvas vs Moodle". 2017. Fecha de consulta: 9 de Enero de 2018. URL: https://www.g2crowd.com/compare/ canvas-vs-moodle

[32] V. Martínez de la Cruz. "Algo Sobre nubes". 2013. Fecha de consulta: 19 de Abril de 2017. URL: http://vmartinezdelacruz.com/ algo-sobre-nubes/

[33] Kamat. "Implanta Navision en local o en la nube". E-Kamat Software de Negocios. 2017. Fecha de consulta: 9 de Enero de 2018. URL: https://www.ekamat.es/navision/local-cloud. php 
[34] I. Walsh. "Que es el cloud computing y que puede aportarnos". 2016. Fecha de consulta: 8 de Enero de 2018. URL: http://www.wetcom. com/content/que-es-el-cloud-computing/
[35] beServices. "Tendencias de Cloud Computing para el 2019”. 2018. Fecha de consulta: 8 de Marzo de 2018.URL: https://www.beservices.es/ cloud-computing-tendencias-2019-n-5357-es 